Western University

Scholarship@Western

Health Studies Publications

Health Studies Program

$1-2009$

\title{
Increasing Capacity for Knowledge Translation: Understanding How Some Researchers Engage Policy-makers
}

Anita Kothari

The University of Western Ontario, akothari@uwo.ca

Lynne McLean

Nancy Edwards

Follow this and additional works at: https://ir.lib.uwo.ca/healthstudiespub

Part of the Medicine and Health Sciences Commons

Citation of this paper:

Kothari, Anita; McLean, Lynne; and Edwards, Nancy, "Increasing Capacity for Knowledge Translation: Understanding How Some Researchers Engage Policy-makers" (2009). Health Studies Publications. 8.

https://ir.lib.uwo.ca/healthstudiespub/8 


\title{
INCREASING CAPACITY FOR KNOWLEDGE TRANSLATION: UNDERSTANDING HOW SOME RESARCHERS ENGAGE POLICY-MAKERS
}

\begin{abstract}
The potential for research to influence policy, and for researchers to influence policy actors, is significant. The purpose of this qualitative study was to explore the experiences of health services researchers engaging in (or not able to engage in) policy-relevant research. Semistructured telephone interviews were completed with 23 experienced researchers. The results paint a complex and dynamic picture of the policy environment and the relationship between government officials and academic researchers. Elements of this complexity included diverse understandings of the nature of policy and how research relates to policy; dealing with multiple stakeholders in the policy-making process; and identifying strategies to manage the different cultures of government and academia.
\end{abstract}

Key Words: knowledge translation, evidence-based policy-making 


\section{INTRODUCTION}

Public policy-making has been described as action (or non-action) by political actors in relation to a social problem. Older models of policy-making, based on a rational approach to making decisions, suggest a linear process whereby a policy problem is identified, possible solutions to the problem are evaluated and a solution is selected and implemented (Mintz et al., 2006). In this tradition, policy-making is confined and defined by civil servants and politicians - formal policy actors. In more recent years, models of policy-making have acknowledged other actors' roles in the policy process, such as the media, public interest groups, or networks and coalitions (Mintz et al., 2006). In particular, researchers, both inside and outside of government, have played stronger roles as technical advisors to complex policy files.

The best case scenario is that this technical advice is based on the findings of research studies. Research is a broad category, encompassing such things as surveys, evaluations, or needs assessments. Hollander and Prince (1993) studied the analytic functions carried out by in-house government groups providing research information. They categorized input to policy-making as falling on a continuum of: forward-looking activities (policy formulation/analysis and planning); ongoing activities for organizational maintenance (internal audit, operational review); and technical and evaluative activities (research, evaluation). Indeed, the input of research into policy determination is one of the components of policy capacity of any government system (Rasmussen, 1999). Even policy management functions are thought to require theoretical research, applied research, statistics and quantitative modelling, environmental scanning, trends analysis and forecasting, policy analysis, and program design, implementation, monitoring, and evaluation (Anderson, 1996). The potential for research to influence policy, and for researchers to influence policy actors, is significant. This interplay needs to be better understood to nurture a culture of evidence-based policy-making.

The concept of knowledge translation provides a lens through which to examine the relationship between researchers and policy actors. Knowledge translation is an interactive exchange of knowledge between researchers and research users - in this case, policy-makers or decision-makers (Kiefer et al., 2005). Reviews of studies demonstrate that face-to-face interaction is vital for the uptake of research and knowledge (Innvaer et al, 2002), yet there is minimal empirical guidance about effective strategies that can be used to bring these two communities closer together (Mitton et al., 2007). A further complication is that policy decisions are determined by a variety of distinct pieces of knowledge, including past experiences, beliefs, values, skills, resources, legislation, protocols, patient preferences and research results (Dobbins, 2007). Policy-makers have lamented the fact that researchers fail to appreciate this policy context, which perhaps contributes to the inability to bring research into the policy-maker's domain.

The purpose of this qualitative study was to explore the experiences of researchers engaging in (or not able to engage in) policy-relevant research, with specific reference to health services research, defined as a multidisciplinary field that examines how social factors, financing systems, organizational structures and processes, health technologies and 
individual behaviours affect access to health care, the quality and cost of health care and health (Lohr \& Steinwachs, 2002). We try to identify how and why some researchers are able to maintain contacts within government, or are considered "experts" by government. This paper is intended for policy units that are being encouraged to become more evidencebased in their activities, and for researchers wanting to become more involved in policyrelevant research or with decision-makers.

The Use of Research in Policy-Making

Previous research has focused on the use of research in policy-making from either the science "push" or policy "pull" direction. Science push refers to efforts to disseminate research findings through traditional passive means, such as through scientific journals; this method has demonstrated little uptake by non-academics (Lomas, 1997). Another approach is to carry out more targeted distribution efforts. For example, researchers are encouraged to consider how to frame the research message in relation to a specific audience (Knott and Wildavsky, 1980; Lavis et al., 2003). As well, summaries of systematic reviews or primary research, accompanied by recommendations for policy, are recommended for more effective utilization of research (Dobbins et al., 2007). The science push model is characterized as a linear, sequential model, where the research process and findings are generated (in isolation) by researchers, with an underlying assumption that the science will speak for itself or some consideration may be given to "getting the word out."

Science pull, on the other hand, refers to those occasions when research is commissioned or requested from government. Counter-intuitively perhaps, the application of research is not always guaranteed in these situations either (Kothari et al, 2005), perhaps owing to the fact that research findings may not be in a usable form for policy-makers or may be irrelevant for the policy problem at hand. Landry and colleagues (2001) have critiqued this model on the basis that it ignores the broad array of knowledge types that might be used in policy-making.

More recently, there has been increased recognition of the differing contexts associated with researchers and policy-makers. Their unique environments result in different cultures (related to how performance is rewarded; types of decisions to be made; timelines; jargon) or "two different communities" (Caplan, 1979; Lomas, 1997). Increased interactions between the policy-maker and the researcher have been proposed as a way to close the twocommunity gap (Elliot and Popay, 2000; Lavis et al., 2003). Indeed, a systematic review of policymaker's use of research identified two-way communication as the most important facilitator of research (Innvaer et al., 2002). Not only can interaction be used to exchange knowledge about professional norms and expectations (Denis et al., 2003), interactions can help users understand research findings. Early interactions can make the research more responsive to policy-maker needs through the refinement of research questions or contextualization of findings to local needs. Lavis and colleagues (2003) note that partnerships can benefit the decision-making process by providing opportunity for decisionmakers to 'internalize' research evidence over time through constant interaction with researchers. 
The interaction model of knowledge translation has led to the development a number of promising strategies or interventions. Knowledge brokering, or human intermediaries between researchers and policy-makers, are being promoted as a way to capitalize on the interaction view of knowledge translation (Lomas, 2007). Networks are another potential mechanism for bringing decision-makers and researchers in close contact (Kothari et al, 2005). The effectiveness of these and other social interaction-based knowledge translation interventions are still under evaluation. This study contributes to understanding these complex interactions, between researcher and policy-maker, in order to inform subsequent strategies to encourage research uptake and utilization.

\section{METHODS}

A phenomenological theory of inquiry guided this analysis (Creswell, 1998); as such, we sought to understand the role of research in influencing policy, and researchers in influencing policy actors, as experienced by researchers. To this end, the study was designed as a qualitative study using semi-structured interviews. In the interests of sampling a variety of experienced researchers across health services areas and regions of the country engaged in policy relevant research, we decided to use stratified random sampling. Three sampling frames were available to identify established health services researchers:

1) researchers receiving operating and personnel grants from federal health research granting agencies (Canadian Institute for Health Research and Canadian Health Services Research Foundation), as listed on the websites of the granting agencies in January, 2004.

2) researchers receiving joint provincial and federal funding (Ontario Ministry of Health and Long Term Care and Canadian Health Services Research Foundation), as listed on the agency websites in February, 2004.

3) researchers receiving provincial funding (Ontario Ministry of Health and Long Term Care) in February, 2004. This list was provided by Ministry staff.

Researchers receiving funding from these sources were, by nature of the funding, conducting policy-relevant research, and typically had to have been fairly experienced. Further, this provided a mix of federal and provincial funding, and therefore, levels of policy concern.

Participants were selected from each of the sampling frames and invited to participate. We recruited 23 volunteers who met the eligibility criteria, and assessed that we had reached saturation. Further, given the policy-related timelines for the research, it was not possible to continue interviews beyond this, using a purposive snow-balling approach.

Using a semi-structured schedule, the 23 researchers were interviewed by telephone for approximately 45 minutes. Questions examined where the researchers were situated in terms of policy structures and networks, the kind of policy-related research they had done, and for what type and level of policy functions. Researchers were also asked how they formulated policy research questions, what facilitators and barriers they experienced in doing policyrelated research, and for their perceptions of the skills and environmental supports needed by researchers involved in policy-relevant research.

The interviews were audio taped and transcribed. Analysis was supported by QSR N6 software. Using a modified content analysis approach (Holsti, 1969), themes were 
developed from a mix of expected and emerging concepts. While some expected concepts were generated from the literature and previous research, and were examined deductively, others emerged inductively through a more standard thematic process. Relationships among themes were compared, triangulated and tested for disconfirmation by the authors. Findings across data sources were also triangulated. All participants had the option of reviewing their transcripts, and all participants were sent copies of the final reports and invited to comment. None chose to do so.

The University of Ottawa Health Sciences and Science Research Ethics Board granted ethical approval for this research (file H 01-04-01). Participants were asked to either sign an informed consent form, or provide consent over the telephone for the use of the information collected. Permission was also sought to use quotes from interviews.

\section{RESULTS}

\section{Sample Demographics}

Tables $1 \& 2$

Twenty-three participants were interviewed (Table 1), with participants relatively evenly divided among the three sampling frames identified for the study. Although the interviewees were all affiliated with an academic setting, they were diverse in terms of their research focus, and work context (Table 2). With a few exceptions the interviewees were senior researchers. At least eight were directors of research institutes and all but two had PhDs. Their professional backgrounds varied considerably. Approximately half had professional training specific to health - medicine, nursing, physiotherapy, and pharmacy - and a few were still practising clinicians. Two were teaching health policy related courses in academic settings and one was the executive director of a health service organization. Over half did some private consulting.

\section{Findings}

\section{A. What is policy-related research?}

Interviewees shared a broad consensus that "policy-related research" is research that has relevance to policy-makers. However, many were less clear about how a "policy" is defined and how the evidence from research influences policy decisions. Even participants with many years of research that closely linked to policy issues found "policy" and "policymaking" to be elusive concepts.

[Policy-related research] means a lot of things...When I look at some of the people doing outcome evaluation work and management work, the policy impact of their work has been enormous... and yet their work, if you want to define it by discipline is not policy.

Health policy is made at numerous levels...I see health policy very much as a continuum from patient through to the macro kind of Ministry of Health [policy]. 
Several interviewees raised issues concerning the impact of research evidence on policy decisions - both whether and how evidence influences decisions. The measure of success for one experienced researcher was that decision-makers were aware of the relevant research, whether or not it influenced the decisions made. This person and others felt an important element in the preparation of health policy researchers was their understanding that research evidence is only one of many influences on policy-makers.

I don't expect policy decisions to be evidence based, but I will have failed if the evidence that is generated as a result of my efforts isn't somehow at least taken note of in the policy-making decisions. It's the difference between science and politics.

A number of different kinds of information were associated with "policy-related/policyrelevant" research, including:

- Research that informs all stages of the policy cycle, from agenda setting or defining the critical questions to evaluation or implementation.

- Research that helps to explain or describe the policy decision-making process.

- Research undertaken in response to specific questions by policy-makers.

- Research informing organizational issues such as guidelines affecting individual patients or policies within institutions.

- Research informing macro issues involving legislative changes and regulations of the government.

- Research with implications for change in large systems or groups in society.

Policy-related research was viewed by some as being knowledge building along a continuum, with learning at all stages being important to inform other levels of decisionmaking. For example, basic clinical knowledge informs best practices, and knowledge of institutional best practices helps to inform systems design. A few respondents regretted the lack of communication among researchers focusing on basic science or clinical issues and those focusing on policy, or considered the current focus on policy research as taking resources from clinical research.

I look at research very much on a continuum, so even the basic work that we're doing in the laboratory...[may have] broad implications...In terms of it being directly related to policy, it's not, but it would suggest that we need to do something at the policy level to make sure that these [services] are accessible. So it's not direct...Everything is related to everything.

Finally, a small number of respondents thought that any research might be policy-related, with its relevance being determined through application, rather than by the intention of the research or by whom it was commissioned. In their opinion, predicting relevance was difficult. 
In addition to attempting to articulate what "policy-relevant" research meant to them, several interviewees made more general comments on the nature of "policy" and how policies are developed. They saw policy issues as broader than government, and other bodies as helping to define and use policy-related research, as well as having an impact on policy directions. Large stakeholder groups (e.g. lobby groups, service providers, consumers, professional associations) were identified. One interviewee identified the "health policy industry" itself as a special interest group influencing allocation of resources.

The complexity of the interaction of multiple factors in determining policy decisions, including values and perceptions of values, and the reality that policy decisions are ultimately a matter of political choice were noted by several interviewees.

One never knows what's going to be relevant next to the policymakers and the health care field... what's relevant today may not be relevant in the future... and depending on what it is, what policymakers need, may or may not be research.

The quote also illustrates the shifting landscape against which policy decisions need to be made.

\section{B. How are policy-related research questions developed?}

Interviewees described various paths by which they came to define specific research questions. About half of the researchers discussed multiple and interconnected paths for research questions, an equal number reported a single process. Most of these mentioned the questions originating from policy-makers. While the issues and directions of their research were influenced by numerous factors, they emphasized that they alone were ultimately responsible for the final research questions and design.

The formulation of my research is one that comes from me, comes from me looking around at the health care system and seeing where there's a need.

We defined the questions; they [government people] brought the issue to us.

You don't often know sometimes that your policy, your research questions are being shaped by your interactions with policy communities, but in fact, I think they probably are. Sometimes it's a key comment that comes up in a meeting somewhere...

Respondents noted that previous work (with respect to methodology, issue expertise, skills) often brought the researchers to the attention of decision makers, who contacted them with issues requiring investigation. These ongoing or opportunistic discussions with government officials helped refine questions. 
...being on that particular [government] committee, I've become aware of what the issues are... what their business plan is, where their focus is.

The shape of the research was initiated on our part, but the opportunity came because they had a very specific call where we could take this question and follow up with it.

In many cases, long term partnerships with not-for-profit or community organizations help to set research priorities. Sometimes iterative processes with input from a variety of stakeholders including any or all of policy-makers, service providers and consumers, contribute to policy-relevant questions.

So we start with a problem that is presented by the consumer or by service providers...Then, we, as researchers, look at what the problems seem to be, do some pilot work around that, and then actually develop a research question that will be framed in a way that will be relevant across more than just that research question...so from this study - whether or not physicians uptake this information that we're giving to them - we will know a lot about how to present information on other topics as well.

A few interviewees felt that, in their experience, research funded provincially tends to have a more specific/targeted focus than nationally funded research. Also, available funding frequently played a role, less in defining an issue than in framing how an issue was presented (also see section C). On the other hand, at least one person commented on the difficulty of tackling issues not seen as a current priority by policy-makers.

I think for some of those people who want to be ahead of the pack, it becomes much harder because your work is shaped by ...what's immediately everybody's concern.

\section{The Fit between Policy-Related Research and the Academic Environment}

The research skills developed and refined in academia, the freedom to pursue inquiries based on the researcher's passions and curiosities (particularly for tenured faculty), and a supportive infrastructure were highlighted as benefits of research in an academic environment. The peer-review process was widely recognized as important to ensuring rigour in applied research, but also mentioned frequently as a challenge with policy-related research. The principles of academic freedom and researcher independence were stressed as important to the credibility of the research by several interviewees. Related to their independence was their right to have the final say in the development of valid and reliable research designs, recognition of intellectual property rights, and input into defining research questions in ways meaningful to the researcher as well as the policy-maker.

The continuity and longevity of the research enterprise provided in a non-government setting was also noted as a benefit. Several interviewees, from both private and academic 
organizations, commented on the benefits to their current work of having accumulated a large data base and/or body of knowledge over a period of time.

Several participants also mentioned the importance of the infrastructure supporting good research. Several researchers affiliated with large institutions and/or research units remarked that the presence of skilled colleagues to provide stimulation and a range of skill sets for specific projects was beneficial. This allowed for greater capacity for flexibility enabling the unit to respond more effectively to the short time frames frequently demanded by governments.

I think size is important, because to keep the applied focus together with the academic, you have to have some flexibility... Everybody is always in a hurry, so my capacity to be responsive is better when I have a larger group of individuals to draw on.

We are very fortunate within our organization that we have a branch of the organization that is focused very much on community development. We also have a policy group within senior management of the organization that works closely with government. We have mechanisms within our organization both to relate to fairly senior levels of the Ministry, and also to work with any program, kind of bottom up.

As well, established resources helped ensure continuity of support staff such as junior researchers and research coordinators which in turn contributed to the quality and quantity of research. For example, a critical mass of staff affords the flexibility to mix "have to do" projects with "want to do" projects that sustain the interest and enthusiasm of researchers

On the other hand, interviewees were virtually unanimous about the different cultures of academia and government, and the challenges these posed. Challenges to doing policyrelated research within an academic community included the incompatibility of the university reward system with government needs and expectations; lack of time and lack of recognition of the time needed to be an effective policy researcher; and the size and complexity of universities.

Many factors contributed to the time challenge of those doing policy-related research. Time was needed for interaction with policy-makers as well as other stakeholders. Demands to be involved in general committee work both within the university and the community seemed to be increasing. Insufficient funding for emerging health policy researchers was also seen as a problem. More career scientist awards, doctoral and post doctoral fellowships and other awards would provide salaries for emerging policy scholars who could assist the current cohort of senior researchers, relieving some of their time challenges. The desire, and need, to go beyond the research report and do knowledge translation work was another time factor. Finally, cross/multi-disciplinary work, the norm in policy-related research, was also time intensive. 


\section{The Fit Between University-Based Research and Government Structures and Practices}

Researchers noted a number of government structures and practices as having an impact on their capacity to do effective policy-related research. Individuals spoke positively about their experiences with opportunities for close, regular communication with policy-makers. For example, events sponsored by the provincial and federal governments to facilitate dialogue among researchers working on the same issue, and between researchers, policy-makers and community stakeholders with common concerns.

Participants also identified leadership from senior officials in defining a broad research agenda on an important issue. This leadership came from major funding sources as well as government decision makers.

However, many interviewees spoke of the challenges inherent in government that had a negative impact on their policy-related research. The single issue mentioned most frequently was the short, often unrealistic, time frames of government. Governments took too long to make decisions about allocating funds for research, whether contract or peerreviewed, and, particularly for contract research, once a decision was taken, they left too little time for quality work.

One of the challenges...is that within government and with other people you're dealing with, they're pressured to deal with something "today". ... They need something today and there may not be the time to really respond to that properly.

The frequency of a crisis orientation was another time problem. Researchers observed that policymakers often seemed to be "fighting fires". This observation was not a critique of individual policymakers, for whom several people expressed high respect, but of government operations generally.

What I have experienced with policy-makers at all levels...is incredible turn over and .... a short attention span. I don't mean that in a negative way, I just mean that they are moving from crisis to crisis, or problem to problem, that's the nature of what they do.

Short funding cycles also reflected the government's time frame, but not that of the researchers. Project funding and annual grants from government inhibited the building of research teams that need several years to mature.

Researchers identified two common practices of government that they found frustrating in terms of policy-related research. Frequent movement of government personnel made it difficult to build the mutual understanding, communication and trust needed for the most effective collaboration.

Last year we finally had a situation where the Ministry staff has been stable. The frustration prior to that would be that you could literally in a four week period of time be talking to somebody else about 
something and they didn't even know that the question had been asked. That was a very big problem.

Excessive concern over secrecy and confidentiality was an additional concern expressed by a couple of participants. This pertained to government data bases and, less frequently, to confidentiality requirements related to reports produced. Access to government records, data bases, and even personnel were quite a concern of the emergent researchers who may not as yet have established a track record of trust with the government groups they have worked with.

Oh yeah there's a lot of barriers, when you look at something like, access to records. It, like at every level it's closed, you don't have access to anything.

Several researchers also commented on the lack of feedback on their work. They received no information about who read a report or what action, if any, it might have influenced.

Finally, participants made a couple of comments about the challenges for researchers of the ongoing federal/provincial jurisdictional conflicts. The different roles of the federal and provincial governments in Canada's health care system had resulted in lack of coordination of policy-related research and/or gaps in research where neither party wanted to assume responsibility. Federal/provincial political sensitivities added one more level of complexity for researchers trying to understand the world of policy-makers.

A huge challenge for me is the split in Canada between products being regulated at a federal level and practices being regulated at a provincial level. Because that's very artificial in the real world of health care. And so often our research will span both but then neither feel they really want to fund it or support it and so I end up taking it apart and rewriting things so that they have a spin one way or another which is a big waste of time in a lot of cases.

\section{E. Opportunities for Multi-Bridging}

A number of interviewees described how working with policy-makers helped them build better relationships and increased their understanding of the policy-making world. While the contrasting cultures of the two groups presented challenges for all parties, many strategies were suggested to bridge the two worlds.

I'm slowly trying to get my head around [what policy is]. That's been one of the neat things [in] a really interesting partnership because they learn to look at things the way we look at them a little bit, and vice versa.

A number of interviewees stated that they had limited contact with government policymakers and that their work focused primarily on what they perceived as non policy-related questions (for example, patient care). However, the majority described a wide range of settings and opportunities they had had for working with government contacts. 
Having built a relationship with these people [decision-makers], it gives me a lot of insight into their world, so I am able to predict in a lot of ways the things that are going to come up.

Personal relationships built over time played an important role for several individuals. The primary contacts for another researcher were a couple of individuals who had had long-term careers within the government department he had dealt with and with whom he had a long history of collaboration. Still others made a point of participating in events - conferences, round table discussions, and symposia - where they would have opportunities to meet new government contacts and interact with policy-makers with whom they had already worked. A few people described positive shifts in ways of relating over time. Infrequent meetings were replaced with regular face-to-face contact for joint discussion and planning and more frequent telephone and e-mail contact.

I really don't think we can underestimate the importance of a personal relationship between the people in the policy environment and the researchers. I'm not talking about friendship, just relationships. Do you know each other? Do you trust each other? Have you worked on enough projects to feel confident that your information won't be misconstrued...?

Several researchers noted that their work on government committees and advisory groups provided an effective venue for learning about issues, becoming more aware of different perspectives on issues, identifying concerns that could become a research question and disseminating their research.

It [working with decision-makers on policy committees] gives you an idea of the culture and mindset, what concerns are around there [government].

A few interviewees noted that funders increasingly demanded the participation of policymakers in the research process. In general, this was welcomed, although time pressures on decision makers could mean their participation was limited. One researcher noted that her policy-maker partner had not been seen since the first meeting.

"Working together" for many of the researchers interviewed meant more than working with government decision-makers. Their research activities were linked to a wide range of stakeholders, including non-governmental groups (including advocacy groups), service providers and consumer groups. The following observation was in the context of work with institutional decision-makers.

I really don't think [having an influence on decision-makers] is about simply taking the results and doing something. It's actually through the process of engaging with decision-makers and research that you really have a greater chance of changing the way they do things. 
I think you involve many different stakeholders. Ultimately any of these things that we do has to make a change in the lives of the people.

Several researchers commented on the active role that non-governmental and community groups played in their work. For most of them, this led to their research being used for advocacy purposes. While they were reconciled to this, it can be a concern as indicated in the following comment.

I sometimes struggle with whether one can really remain objective and neutral in one's positions on things because I end up over time having to take certain advocacy stances around particular positions and then worry that people may not be impressed.

\section{F. Personal Factors - Making it Happen}

When asked what had contributed to their own successful work in policy-related research and what advice they would give to researchers new to this field, many researchers described a combination of personal and professional qualities.

Having good research skills and producing quality results were seen as the basis for good policy-related research. In different ways, a number of researchers alluded to the importance of their reputation for being able to deliver results. Many of the researchers interviewed were recognized as "experts" in a particular methodology, or in relation to a particular field. Their reputation was an important component of the "trust" they felt was required for successful relationships with policy-makers.

Beyond technical research skills, a few people commented on the importance of broader conceptual skills such as the ability "to see links between big questions and how short-term projects can be linked to longer-term issues" and "to draw policy-relevant results from broader results of research."

Inherent personality traits were also recognized as an asset to policy-related research work. Good social skills were most important. People need a special kind of personality "to work in an interactive mode in a political context." Networking, sitting on boards and committees and generally knowing lots of people were seen as essential by many interviewees. Doing this well depended on having good social skills.

Sitting on the board has definitely opened my eyes to other avenues and other types of research questions that I may not have asked otherwise.

Make sure you [new researchers] understand not only good science, but also build skills in terms of processes and interactions with decision-making partners. 
Communication skills were also seen as extremely important in a number of facets of policy-related research. Several participants mentioned the challenges, as well as the importance, of involving and communicating with many different stakeholders government policy-makers, institutional policy-makers and community groups. One person involved in survey research spoke of the need to move beyond the tables and charts and put results in a "story" that communicates with policy-makers.

We always want to be interesting and we always want to have a story to tell. Telling stories is a really important part of research.

Two other personal qualities identified were humility and an element of entrepreneurism. Several interviewees commented on the need for researchers to be realistic about the limited role of research in policy decision-making and a few mentioned the need to "keep your ego in your pocket".

There are a lot of factors that go into the policy environment...I think researchers would probably do well to learn a lot more about them. You learn these things over time, and not to get ticked off when they ask you to spend a lot of time on something, then it might become a really small part of what they're doing or get watered down.

The entrepreneurial aspect related to people's ability to identify and use opportunities to further their own research agenda. Although the primary reference was to knowing how to capitalize on funding opportunities, the comments also referred to understanding how to work with situations and opportunities with people to gather knowledge and present one's own research agenda. Emergent researchers discussed also the need for confidence in going forward and taking the risk, trying to meet the challenges of this type of research. They further mentioned a need for passion and motivation.

The final personal factor mentioned was having previous experience as managers or policymakers. Several researchers who had worked as either institutional managers or within government noted that this experience gave them both a better understanding of the issues faced by policy-makers and possibly increased their credibility in their interaction with policy-makers.

Maybe my strong understanding of the need for policy-makers and practitioners to have evidence is that I didn't work in the academic group my whole life. I came to the PhD having been a [health services manager] and a management consultant.

\section{DISCUSSION}

The results of the interviews paint a relatively complex and dynamic picture both of the policy environment and the relationship between government officials and academic researchers. Elements of this complexity included: 
- defining the nature of policy and how research relates to policy

- dealing with multiple stakeholders in the policy-making process, and

- finding ways to deal with the very different cultures of government and academia

Most researchers participating in the study recognized the dynamic and political nature of the policy-making process. In their collective experience, policy had many different expressions and many different, although not always obvious, paths potentially linking policy-related research to policy. For some, policy-related research frequently focused on a crisis or short-term problem, lacked a strategic or long-term context, and resulted in reports on which they received little or no feedback. For others, their research was acknowledged and read by people within government, but whether or not had made a difference seemed questionable. Still others spoke of research, not commissioned directly by government, which ultimately did have an impact on provincial policies.

For several researchers, relationships with community groups were more stable over time than those with government officials. These groups were valued partners in defining research priorities, shaping research questions and disseminating research results. Through advocacy, aided by research evidence, some community partners also played a role in influencing policy. Several researchers saw their involvement with community and institutional partners as providing insights into issues that helped inform their work with government officials. Given better opportunities for dialogue, they felt that these insights could make a significant contribution to longer-term planning within government.

The findings confirmed what the current knowledge transfer literature suggests about widely different cultures between government and academia typified by different time frames and reward systems (Coburn, 1998; Crosswaite and Curtice, 1994; Lomas, 2000). Some interviewees experienced this clash of cultures as a tension over control - government's desire to "micro-manage" projects versus the researcher's need to control the research process. For others, it was reflected in unrealistic expectations of the kinds of questions that could be answered and the time needed to answer them. Finally, several noted the lack of recognition by government officials of how policy-relevant research related, or not, to the academic career of the researcher.

The frequently short time frames of government requests versus the longer time frames of the academic community were the challenge most frequently mentioned. Several researchers suggested that the best "match" between the resources of the academic community and the needs of governments for policy-related research would be in areas relevant to medium to long-term planning. Furthermore, they stated that their having a better understanding of government priorities in the medium to long term would inform research requests for shortterm projects, making the results more useful.

Issues related to different reward systems have been well documented in the literature (Hofmeyer et al, 2007; Locock and Boaz, 2005; Lomas 2007). Most researchers recognized the role and legitimacy of peer-reviewed publications as a primary tool for evaluation and advancement within the academic community. Given this reality, they stated that more could 
be done both within universities and within governments to recognize and value policyrelated research.

While not anticipating significant changes in either culture in the short-term, many researchers suggested strategies that might help to bridge the gap, with several emphasizing that building bridges required efforts by both parties to build respect and develop reasonable, mutual expectations. Several thought that the level of understanding between researchers and policy-makers was improving over time as a result of increasing opportunities for interaction. A few researchers felt that increased dialogue would lead, not only to more effective work with individual researchers, but also to synergies as overlapping efforts and interests were revealed.

The strongest theme in the study emerged as the need for ongoing and increased efforts to build a level of understanding between government officials and researchers. On the one hand, the study identified the need for government representatives to make efforts to understand the academic community and to communicate more, and more effectively, about the nature of policy, the policy-process, and the role academic research plays in the process. On the other hand, participants recommended that researchers be proactive in seeking out opportunities for dialogue with policy-makers, including spending time with policy-makers on the job.

\section{Recommendations to support evidence-informed policy}

Based on analysis of the data, the following recommendations are directed to research funders and leaders in the academic community.

1. Funders should consider internal strategies and processes to provide academic researchers with a longer-term, broader context for specific research requests. Given the many stages in the policy development process, these strategies should also address how to engage academic researchers in research and dialogue related to agenda setting and priority setting in their areas of expertise.

2. Funders should develop more explicit definitions of "policy-related research" and, in consultation with the academic community, develop different strategies for working with researchers based on an understanding of the best match between government's needs and the skills, strengths and limitations of academic researchers.

3. Funders should expand their support for emerging scholars - doctoral students, postdoctoral fellows and career scientists - to ensure future skilled policy-related researchers.

4. Given the time needed to build strong, multi-disciplinary research teams and the benefits of mature research programs to informing policy, funders should consider multi-year funding for research programs that provide long-term infrastructure support for researchers.

5. Given the contribution of communication, and consistent and open relationships between researchers and decision-makers in building a policy-relevant program of research, funders should formalize multiple strategies to promote interaction and genuine dialogue between the two communities. These strategies could include: 
- opportunities for young researchers to have temporary placements in government ministries where they could experience first-hand the realities of government decision-making

- events that facilitate dialogue and networking between government \& researchers and among researchers with related interests

- opportunities for formal "cross-cultural" learning involving decision-makers and academic researchers

- briefing sessions for research partners on the dynamics of policy development with reference to the role played by evidence, as well as other factors

- workshops and other formal sessions that contribute to a mutual understanding of the different points of view and working realities of the two communities

6. Given the complexity of health policy-related research, academic institutions should ensure that senior researchers are encouraged and rewarded for mentoring newer researchers entering this field.

7. Given the contribution of many levels of health-related research to policy debate and formation, academic institutions should consider courses on understanding health policy for all scholars involved in health research. Given the complexity of health policy research, this preparation should also include education and experience related to working across disciplines on research projects and programs.

\section{CONCLUSION}

This study was designed to gain an understanding about experienced researchers' perceptions of their capacity to do effective policy-related work. The picture of a successful researcher with a high capacity to do policy relevant work emerging from the study was of someone with excellent research skills, including skills to work with inter-disciplinary teams; ongoing, good connections to policy-makers, both formal and informal; and effective social and communication skills. These attributes helped the researcher move between the academic and government communities, engaging with community partners, and developing a program of research that met personal, academic and government needs. This successful researcher would build iteratively on government suggestions and their own interests, and work both strategically and opportunistically to match short and longer-term funding opportunities with the directions they have chosen.

Acknowledgement

Dr. Anita Kothari is partially supported by a career scientist award from the Ontario Ministry of Health and Long-Term Care. Dr. Nancy Edwards holds a Chair in multiple interventions in Community Health Nursing from the Canadian Health Services Research Foundation, the Canadian Institutes of Health Research and the Ontario Ministry of Health 
and Long-term Care. The work reported here was supported by the Ontario Ministry of Health and Long Term Care through a Systems-Linked Research Grant to the Community Health Research Unit. The opinions expressed here are those of the authors. Publication does not imply any endorsement of these views by either of the participating partners of the CHRU, or by the Ontario Ministry of Health and Long-Term Care. 


\section{References}

Anderson, G. (1996) "The new focus on the policy capacity of the federal government." Canadian Public Administration/ Administration Publique du Canada, vol. 39, no. 4, pp. 469-488.

Caplan, N. (1979) "The two-communities theory and knowledge utilization." American Behavioral Scientist, vol. 22, no. 3, pp. 459-470.

Coburn, A.F. (1998) "The role of health services research in developing state health policy." Health Affairs, vol. 17, no. 1, pp. 139-151.

Cresswell, J.C. (1998) Qualitative Inquiry and Research Design; Choosing Among Five Traditions, Thousand Oaks, California: Sage Publications Inc.

Crosswaite, C. \& Curtice, L. (1994) "Disseminating research results- the challenge of bridging the gap between health research and health action." Health Promotion International, vol. 9, pp. 289-296.

Denis, J.L., Lehoux, P., Hivon, M. \& Champagne, F. (2003) "Creating a new articulation between research and practice through policy? The views and experiences of researchers and practitioners." Journal of Health Services Research \& Policy, vol. 8, no. 4 Suppl2, pp. 44-50.

Dobbins, M., Jack, S., Thomas, H. \& Kothari, A. (2007) “Ontario Public Health Decision Makers' Informational Needs and Preferences for Receiving Research Evidence.” Worldviews on Evidence-Based Nursing, vol. 4, no. 3, pp. 156-163.

Elliot, H. \& Popay, J. (2000) "How are policy makers using evidence? Models of research utilization and local NHS policy making." Journal of Epidemiology and Community Health, vol. 54, pp. 461-468.

Hoffmeyer, A., Newton, M., Scott, C. (2007) "Valuing the scholarship of integration and the scholarship of application in the academy for health sciences scholars: recommended methods", Health Research and Policy Systems, vol. 5, no. 1, pp. 5.

Hollander, M.J. \& \& Prince, M.J. (1993) "Analytical units in federal and provincial governments: Origins, functions, and suggestions for effectiveness." Canadian Public Administration/Administration Publique du Canada, vol. 36, no. 2, pp. 120-224.

Holsti, O. (1969). Content Analysis for the Social Sciences and Humanities. Reading, Mass.: Addison-Wesley Publishing Co.

Innvaer, S., Vist, G.E., Trommald, M. \& Oxman, A.D. (2002) "Health policy-makers' perceptions of their use of evidence: A systematic review." Journal of Health Services Research \& Policy, vol. 7, pp. 239-244.

Kiefer L, Frank J, Di Ruggiero E, Dobbins M, Manuel D, Gully PR, et al. (2005) "Fostering evidence-based decision-making in Canada: examining the need for a Canadian 
population and public health evidence centre and research network." Canadian Journal of Public Health, vol. 96, no. 3, pp.1-20.

Knott, J. \& Wildavsky, A. (1980) "If Dissemination Is the Solution, What Is the Problem ?", Science Communication, vol. 1, no. 4, pp. 537-578.

Kothari, A., Birch, S. \& Charles, C. (2005) "“Interaction" and Research Utilisation in Health Policies and Programs: Does it Work?", Health Policy, vol. 71, pp. 117-125.

Kothari, A., Edwards, N., Brajtman, S., Campbell, B., Hamel, N., Legault, F., Mill, J., Valaitis, R., (2005). "Fostering Interactions: The Networking Needs of Community Health Nursing Researchers and Decision-Makers." Evidence \& Policy: A Journal of Research, Debate and Practice, vol. 1, no. 3, pp. 291-304.

Landry R., Amara N., Lamari M. (2001) "Utilization of social science research knowledge in Canada." Research Policy, vol. 30, pp. 333-49.

Lavis, J.N., Robertson, D., Woodside, J.M., Mcleod, C.B. \& Abelson, J. (2003) "How Can Research Organizations More Effectively Transfer Research Knowledge to Decision Makers;", The Milbank Quarterly, vol. 81, no. 2, pp. 221-248.

Locock, L \& Boaz, A. (2004) "Research, policy and practice - worlds apart?", Social Policy and Society, vol. 3, pp 375-384.

Lohr K.N., Steinwachs D. (2002) "Health services research: an evolving definition of the field." Health Services Research vol. 37, no. 1, pp. 7-9.

Lomas, J. (1997) "Improving research dissemination and uptake in health the health sector: Beyond the sound of one hand clapping." Policy Commentary C-97-1, Hamilton: Canada. McMaster University Centre for Health Economics and Policy Analysis.

Lomas, J. (2000). "Using 'linkage and exchange' to move research into policy at a Canadian foundation.", Health Affairs, vol. 19, no. 3, pp. 236-240.

Lomas, J. (2007). "The in-between world of knowledge brokering." British Medical Journal, vol. 334, no. 7585 , pp. 129-132.

Mintz, E., Close, D. and Croci, O. (2006) Politics Power and the Common Good - An Introduction to Political Science, Toronto, Canada: Pearson Education Canada.

Mitton, C., Adair, C.E., McKenzie E., Patten SB, Waye Perry B. (2007) "Knowledge transfer and exchange: review and synthesis of the literature." The Milbank Quarterly, vol. 85 , no. 4 , pp. $729-768$.

Rasmussen, K. (1999) "Policy capacity in Saskatchewan: strengthening the equilibrium.", Canadian Public Administration/Administration Publique du Canada, vol. 42, no. 3, pp. 331-348. 